\title{
Mixed P-N and As-N Bis-Ylide Palladium Complexes: Cooperative Intramolecular Interactions, Conformational Preferences and C-H Bond Activations
}

Elena Serrano, ${ }^{a}$ Cristina Vallés, ${ }^{\mathrm{b}}$ Jordi J. Carbó, ${ }^{\mathrm{c}}$ Agustí Lledós, ${ }^{\mathrm{d}}$ Tatiana Soler, ${ }^{\mathrm{e}}$ Rafael Navarro, ${ }^{a}$ and Esteban P.Urriolabeitia. ${ }^{a}$

\section{Supporting Information}

Dr. Esteban P. Urriolabeitia (corresponding author): Universidad de Zaragoza-C.S.I.C., Plaza de San Francisco s/n, E-50009 Zaragoza (Spain). Fax: (+34) 976761187. E-mail: esteban@unizar.es 


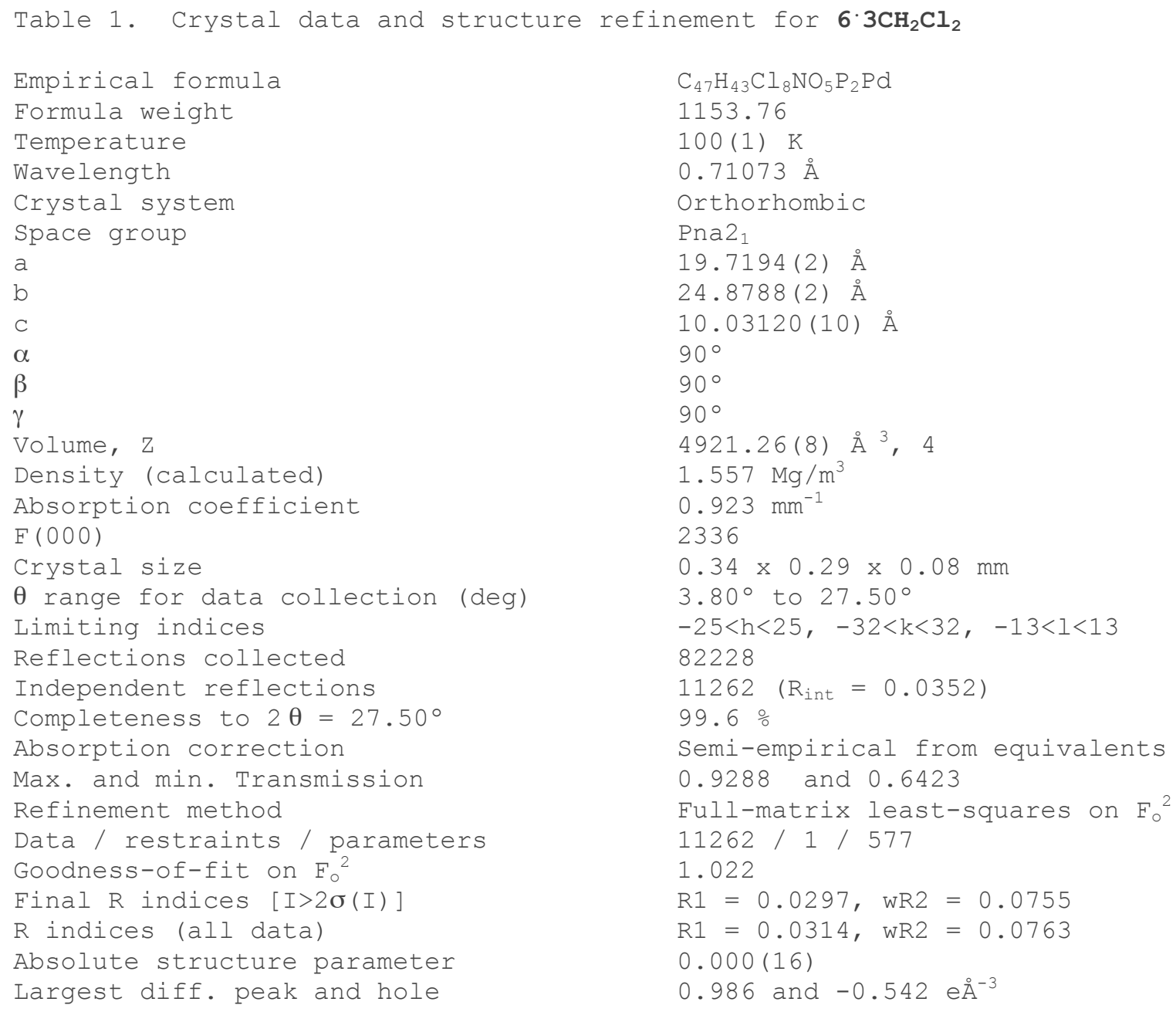


Table 2. Atomic coordinates $\left[\mathrm{x}^{4} 0^{4}\right]$ and equivalent isotropic displacement parameters $\left[\AA^{2} \times 10^{3}\right]$ for $6 \cdot 3 \mathrm{CH}_{2} \mathrm{Cl}_{2}$. U (eq) is defined as one third of the trace of the orthogonalized $\mathrm{U}_{i j}$ tensor.

\begin{tabular}{|c|c|c|c|c|}
\hline & $x$ & y & z & $\mathrm{U}(\mathrm{eq})$ \\
\hline $\mathrm{Pd}(1)$ & $7172(1)$ & $5815(1)$ & $6722(1)$ & $14(1)$ \\
\hline $\mathrm{Cl}(1)$ & $6109(1)$ & $6165(1)$ & $6098(1)$ & $20(1)$ \\
\hline$C(1)$ & $8247(1)$ & $6246(1)$ & 6595 (3) & $18(1)$ \\
\hline$O(1)$ & $8599(1)$ & $6350(1)$ & $5611(2)$ & $20(1)$ \\
\hline C (2) & $7688(1)$ & $6573(1)$ & $7112(2)$ & $17(1)$ \\
\hline $\mathrm{P}(1)$ & $7538(1)$ & $7193(1)$ & $6316(1)$ & $17(1)$ \\
\hline$C(3)$ & $7098(1)$ & $7120(1)$ & $4771(3)$ & $20(1)$ \\
\hline C (4) & $7367(2)$ & $6781(1)$ & $3791(3)$ & $25(1)$ \\
\hline C (5) & $7007(2)$ & $6696(2)$ & $2622(3)$ & $33(1)$ \\
\hline$C(6)$ & $6394(2)$ & $6947(1)$ & $2430(3)$ & $33(1)$ \\
\hline C (7) & $6122(2)$ & $7278(1)$ & $3397(3)$ & $31(1)$ \\
\hline C (8) & $6472(2)$ & $7369(1)$ & $4576(3)$ & $23(1)$ \\
\hline$C(9)$ & $8325(1)$ & $7541(1)$ & $6012(3)$ & $21(1)$ \\
\hline$C(10)$ & $8445(2)$ & $7797(1)$ & $4806(3)$ & $29(1)$ \\
\hline C (11) & $9066(2)$ & $8045(1)$ & $4590(4)$ & $37(1)$ \\
\hline$C(12)$ & $9560(2)$ & $8033(1)$ & $5570(4)$ & $35(1)$ \\
\hline$C(13)$ & $9437(1)$ & $7779(1)$ & $6790(4)$ & $31(1)$ \\
\hline$C(14)$ & $8816(2)$ & $7534(1)$ & 7005 (3) & $25(1)$ \\
\hline$C(15)$ & $7042(1)$ & $7600(1)$ & $7429(3)$ & $20(1)$ \\
\hline$C(16)$ & $7179(2)$ & $8147(1)$ & $7529(3)$ & $24(1)$ \\
\hline C (17) & $6816(2)$ & $8462(1)$ & $8420(3)$ & $28(1)$ \\
\hline C (18) & $6312(2)$ & $8233(1)$ & $9196(3)$ & $28(1)$ \\
\hline C (19) & $6166(2)$ & $7689(1)$ & $9079(3)$ & $28(1)$ \\
\hline C (20) & $6535(2)$ & $7370(1)$ & 8196 (3) & $24(1)$ \\
\hline C (21) & $8184(1)$ & $5709(1)$ & $7243(3)$ & $16(1)$ \\
\hline $\mathrm{N}(1)$ & $8633(1)$ & 5301 (1) & $6723(3)$ & $17(1)$ \\
\hline C (22) & $9012(1)$ & $5008(1)$ & $7583(3)$ & $22(1)$ \\
\hline C (23) & $9448(2)$ & $4622(1)$ & 7125 (3) & $29(1)$ \\
\hline C (24) & $9507(2)$ & $4532(1)$ & $5776(4)$ & $33(1)$ \\
\hline C (25) & $9121(2)$ & $4835(1)$ & $4904(3)$ & $29(1)$ \\
\hline$C(26)$ & $8685(1)$ & $5219(1)$ & $5392(3)$ & $21(1)$ \\
\hline P (2) & $6851(1)$ & $4934(1)$ & 6591 (1) & $14(1)$ \\
\hline C (27) & $6786(1)$ & $4692(1)$ & $4881(3)$ & $17(1)$ \\
\hline C (28) & $6459(2)$ & $4208(1)$ & $4600(3)$ & $20(1)$ \\
\hline C (29) & $6416(2)$ & $4024(1)$ & 3295 (3) & $25(1)$ \\
\hline C (30) & $6702(2)$ & $4325(1)$ & $2268(3)$ & $26(1)$ \\
\hline C (31) & $7022(2)$ & $4804(1)$ & $2548(3)$ & $25(1)$ \\
\hline C (32) & $7063(1)$ & $4993(1)$ & 3852 (3) & $20(1)$ \\
\hline C (33) & $7412(1)$ & $4471(1)$ & $7452(2)$ & $17(1)$ \\
\hline C (34) & $7582(1)$ & $4594(1)$ & $8778(3)$ & $18(1)$ \\
\hline C (35) & $7974(2)$ & $4245(1)$ & $9519(3)$ & $22(1)$ \\
\hline C (36) & $8201(2)$ & $3770(1)$ & 8966 (3) & $27(1)$ \\
\hline C (37) & $8042(2)$ & $3646(1)$ & $7650(3)$ & $29(1)$ \\
\hline C (38) & $7649(1)$ & $3996(1)$ & $6897(3)$ & $23(1)$ \\
\hline C (39) & $6017(1)$ & $4765(1)$ & 7270 (3) & $17(1)$ \\
\hline$C(40)$ & $5931(1)$ & $4456(1)$ & $8423(3)$ & $19(1)$ \\
\hline
\end{tabular}




\begin{tabular}{lrrrr}
$C(41)$ & $5280(2)$ & $4341(1)$ & $8866(3)$ & $25(1)$ \\
$\mathrm{C}(42)$ & $4721(2)$ & $4520(1)$ & $8196(3)$ & $24(1)$ \\
$\mathrm{C}(43)$ & $4804(1)$ & $4836(1)$ & $7060(3)$ & $22(1)$ \\
$\mathrm{C}(44)$ & $5447(1)$ & $4954(1)$ & $6608(3)$ & $19(1)$ \\
$\mathrm{Cl}(2)$ & $8563(1)$ & $5741(1)$ & $1318(1)$ & $27(1)$ \\
$\mathrm{O}(2)$ & $8056(2)$ & $5945(2)$ & $451(3)$ & $74(1)$ \\
$\mathrm{O}(3)$ & $8292(2)$ & $5723(2)$ & $2611(3)$ & $65(1)$ \\
$\mathrm{O}(4)$ & $9131(1)$ & $6099(1)$ & $1360(5)$ & $69(1)$ \\
$\mathrm{O}(5)$ & $8793(2)$ & $5235(1)$ & $852(4)$ & $66(1)$ \\
$\mathrm{C}(45)$ & $8672(2)$ & $7241(2)$ & $1218(5)$ & $54(1)$ \\
$\mathrm{Cl}(3)$ & $8117(1)$ & $7755(1)$ & $701(2)$ & $105(1)$ \\
$\mathrm{Cl}(4)$ & $9508(1)$ & $7435(1)$ & $1189(2)$ & $88(1)$ \\
$\mathrm{C}(46)$ & $5578(2)$ & $5869(1)$ & $9248(3)$ & $28(1)$ \\
$\mathrm{Cl}(5)$ & $5140(1)$ & $6435(1)$ & $9883(1)$ & $34(1)$ \\
$\mathrm{Cl}(6)$ & $6415(1)$ & $5841(1)$ & $9876(1)$ & $29(1)$ \\
$\mathrm{C}(47)$ & $10057(2)$ & $6450(1)$ & $6796(4)$ & $30(1)$ \\
$\mathrm{Cl}(7)$ & $10448(1)$ & $5836(1)$ & $6335(1)$ & $39(1)$ \\
$\mathrm{Cl}(8)$ & $9658(1)$ & $6387(1)$ & $8359(1)$ & $31(1)$ \\
& & & & \\
\hline
\end{tabular}


Table 3. Bond lengths $[\AA]$ and angles $\left[{ }^{\circ}\right]$ for $6 \cdot 3 \mathbf{C H}_{2} \mathbf{C l}_{2}$

\begin{tabular}{|c|c|}
\hline $\mathrm{Pd}(1)-\mathrm{C}(21)$ & $2.080(3)$ \\
\hline $\mathrm{Pd}(1)-\mathrm{C}(2)$ & $2.179(3)$ \\
\hline $\mathrm{Pd}(1)-\mathrm{P}(2)$ & $2.2853(6)$ \\
\hline $\mathrm{Pd}(1)-\mathrm{Cl}(1)$ & $2.3552(6)$ \\
\hline $\mathrm{Pd}(1)-\mathrm{C}(1)$ & $2.379(2)$ \\
\hline$C(1)-O(1)$ & $1.234(3)$ \\
\hline$C(1)-C(2)$ & $1.464(4)$ \\
\hline$C(1)-C(21)$ & $1.491(4)$ \\
\hline$C(2)-P(1)$ & $1.763(3)$ \\
\hline $\mathrm{C}(2)-\mathrm{H}(2)$ & 1.0000 \\
\hline$P(1)-C(3)$ & $1.786(3)$ \\
\hline$P(1)-C(15)$ & $1.797(3)$ \\
\hline$P(1)-C(9)$ & $1.801(3)$ \\
\hline$C(3)-C(8)$ & $1.395(4)$ \\
\hline$C(3)-C(4)$ & $1.399(4)$ \\
\hline$C(4)-C(5)$ & $1.387(4)$ \\
\hline $\mathrm{C}(4)-\mathrm{H}(4)$ & 0.9500 \\
\hline$C(5)-C(6)$ & $1.374(5)$ \\
\hline $\mathrm{C}(5)-\mathrm{H}(5)$ & 0.9500 \\
\hline$C(6)-C(7)$ & $1.381(5)$ \\
\hline $\mathrm{C}(6)-\mathrm{H}(6)$ & 0.9500 \\
\hline$C(7)-C(8)$ & $1.389(4)$ \\
\hline $\mathrm{C}(7)-\mathrm{H}(7)$ & 0.9500 \\
\hline $\mathrm{C}(8)-\mathrm{H}(8)$ & 0.9500 \\
\hline$C(9)-C(10)$ & $1.388(4)$ \\
\hline$C(9)-C(14)$ & $1.389(4)$ \\
\hline$C(10)-C(11)$ & $1.387(4)$ \\
\hline $\mathrm{C}(10)-\mathrm{H}(10)$ & 0.9500 \\
\hline$C(11)-C(12)$ & $1.385(5)$ \\
\hline $\mathrm{C}(11)-\mathrm{H}(11)$ & 0.9500 \\
\hline$C(12)-C(13)$ & $1.397(5)$ \\
\hline $\mathrm{C}(12)-\mathrm{H}(12)$ & 0.9500 \\
\hline$C(13)-C(14)$ & $1.386(4)$ \\
\hline C (13) - H (13) & 0.9500 \\
\hline $\mathrm{C}(14)-\mathrm{H}(14)$ & 0.9500 \\
\hline$C(15)-C(20)$ & $1.385(4)$ \\
\hline$C(15)-C(16)$ & $1.391(4)$ \\
\hline$C(16)-C(17)$ & $1.386(4)$ \\
\hline $\mathrm{C}(16)-\mathrm{H}(16)$ & 0.9500 \\
\hline$C(17)-C(18)$ & $1.384(5)$ \\
\hline $\mathrm{C}(17)-\mathrm{H}(17)$ & 0.9500 \\
\hline$C(18)-C(19)$ & $1.388(5)$ \\
\hline $\mathrm{C}(18)-\mathrm{H}(18)$ & 0.9500 \\
\hline$C(19)-C(20)$ & 1.395 (4) \\
\hline C (19) -H (19) & 0.9500 \\
\hline $\mathrm{C}(20)-\mathrm{H}(20)$ & 0.9500 \\
\hline $\mathrm{C}(21)-\mathrm{N}(1)$ & $1.444(3)$ \\
\hline $\mathrm{C}(21)-\mathrm{H}(21)$ & 1.0000 \\
\hline $\mathrm{N}(1)-\mathrm{C}(26)$ & $1.355(4)$ \\
\hline $\mathrm{N}(1)-\mathrm{C}(22)$ & $1.356(4)$ \\
\hline$C(22)-C(23)$ & $1.368(4)$ \\
\hline
\end{tabular}




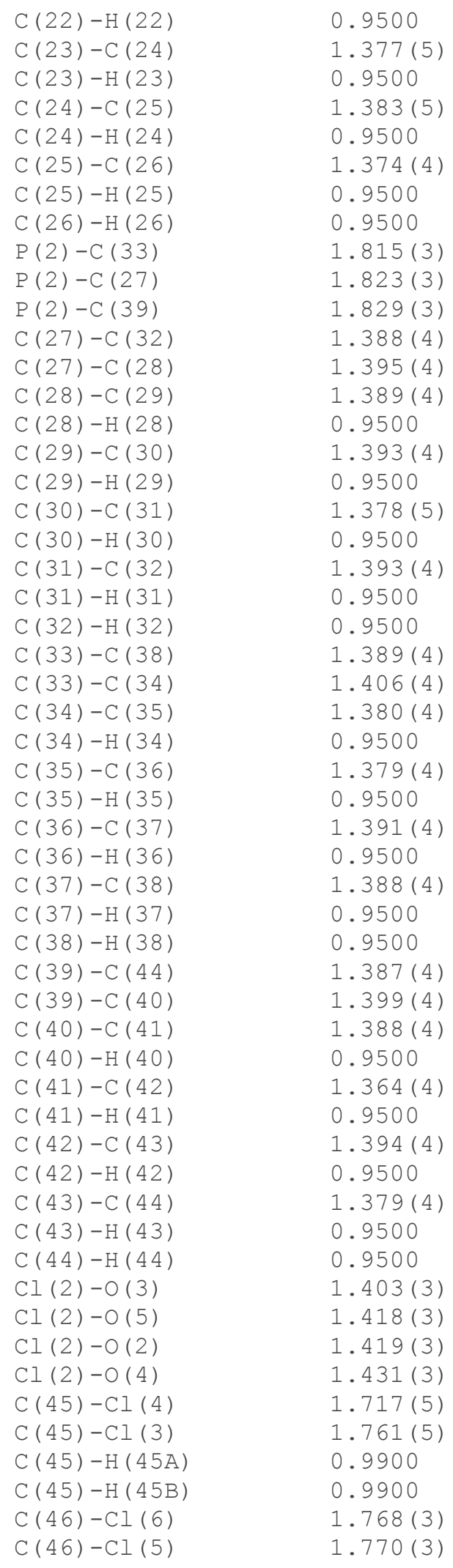




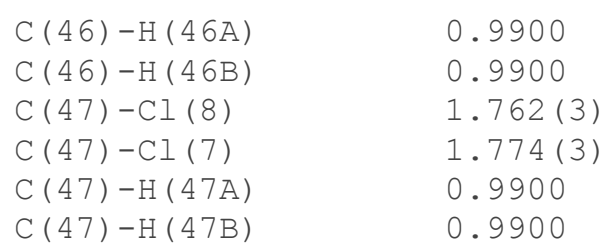

0.9900

0.9900

$1.762(3)$

$1.774(3)$

0.9900

0.9900

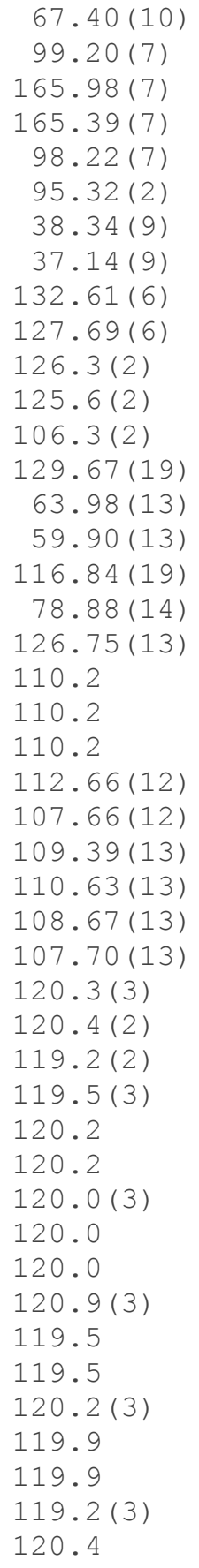




\begin{tabular}{|c|c|}
\hline $\mathrm{C}(3)-\mathrm{C}(8)-\mathrm{H}(8)$ & 120.4 \\
\hline$C(10)-C(9)-C(14)$ & $120.7(3)$ \\
\hline$C(10)-C(9)-P(1)$ & $121.0(2)$ \\
\hline$C(14)-C(9)-P(1)$ & $118.2(2)$ \\
\hline$C(11)-C(10)-C(9)$ & $119.5(3)$ \\
\hline $\mathrm{C}(11)-\mathrm{C}(10)-\mathrm{H}(10)$ & 120.3 \\
\hline $\mathrm{C}(9)-\mathrm{C}(10)-\mathrm{H}(10)$ & 120.3 \\
\hline$C(12)-C(11)-C(10)$ & $120.0(3)$ \\
\hline $\mathrm{C}(12)-\mathrm{C}(11)-\mathrm{H}(11)$ & 120.0 \\
\hline $\mathrm{C}(10)-\mathrm{C}(11)-\mathrm{H}(11)$ & 120.0 \\
\hline$C(11)-C(12)-C(13)$ & $120.7(3)$ \\
\hline $\mathrm{C}(11)-\mathrm{C}(12)-\mathrm{H}(12)$ & 119.7 \\
\hline $\mathrm{C}(13)-\mathrm{C}(12)-\mathrm{H}(12)$ & 119.7 \\
\hline$C(14)-C(13)-C(12)$ & $119.2(3)$ \\
\hline $\mathrm{C}(14)-\mathrm{C}(13)-\mathrm{H}(13)$ & 120.4 \\
\hline $\mathrm{C}(12)-\mathrm{C}(13)-\mathrm{H}(13)$ & 120.4 \\
\hline$C(13)-C(14)-C(9)$ & $120.0(3)$ \\
\hline $\mathrm{C}(13)-\mathrm{C}(14)-\mathrm{H}(14)$ & 120.0 \\
\hline $\mathrm{C}(9)-\mathrm{C}(14)-\mathrm{H}(14)$ & 120.0 \\
\hline$C(20)-C(15)-C(16)$ & $120.3(3)$ \\
\hline$C(20)-C(15)-P(1)$ & $120.3(2)$ \\
\hline$C(16)-C(15)-P(1)$ & $119.4(2)$ \\
\hline$C(17)-C(16)-C(15)$ & $119.9(3)$ \\
\hline $\mathrm{C}(17)-\mathrm{C}(16)-\mathrm{H}(16)$ & 120.1 \\
\hline $\mathrm{C}(15)-\mathrm{C}(16)-\mathrm{H}(16)$ & 120.1 \\
\hline$C(18)-C(17)-C(16)$ & $120.1(3)$ \\
\hline $\mathrm{C}(18)-\mathrm{C}(17)-\mathrm{H}(17)$ & 120.0 \\
\hline $\mathrm{C}(16)-\mathrm{C}(17)-\mathrm{H}(17)$ & 120.0 \\
\hline$C(17)-C(18)-C(19)$ & $120.1(3)$ \\
\hline $\mathrm{C}(17)-\mathrm{C}(18)-\mathrm{H}(18)$ & 119.9 \\
\hline $\mathrm{C}(19)-\mathrm{C}(18)-\mathrm{H}(18)$ & 119.9 \\
\hline$C(18)-C(19)-C(20)$ & $120.0(3)$ \\
\hline $\mathrm{C}(18)-\mathrm{C}(19)-\mathrm{H}(19)$ & 120.0 \\
\hline $\mathrm{C}(20)-\mathrm{C}(19)-\mathrm{H}(19)$ & 120.0 \\
\hline$C(15)-C(20)-C(19)$ & $119.6(3)$ \\
\hline$C(15)-C(20)-H(20)$ & 120.2 \\
\hline $\mathrm{C}(19)-\mathrm{C}(20)-\mathrm{H}(20)$ & 120.2 \\
\hline $\mathrm{N}(1)-\mathrm{C}(21)-\mathrm{C}(1)$ & $115.0(2)$ \\
\hline $\mathrm{N}(1)-\mathrm{C}(21)-\mathrm{Pd}(1)$ & $125.83(17)$ \\
\hline $\mathrm{C}(1)-\mathrm{C}(21)-\mathrm{Pd}(1)$ & $81.76(15)$ \\
\hline $\mathrm{N}(1)-\mathrm{C}(21)-\mathrm{H}(21)$ & 110.3 \\
\hline $\mathrm{C}(1)-\mathrm{C}(21)-\mathrm{H}(21)$ & 110.3 \\
\hline $\mathrm{Pd}(1)-\mathrm{C}(21)-\mathrm{H}(21)$ & 110.3 \\
\hline$C(26)-N(1)-C(22)$ & $120.3(2)$ \\
\hline$C(26)-N(1)-C(21)$ & $120.6(2)$ \\
\hline$C(22)-N(1)-C(21)$ & $119.1(2)$ \\
\hline$N(1)-C(22)-C(23)$ & $120.7(3)$ \\
\hline $\mathrm{N}(1)-\mathrm{C}(22)-\mathrm{H}(22)$ & 119.6 \\
\hline $\mathrm{C}(23)-\mathrm{C}(22)-\mathrm{H}(22)$ & 119.6 \\
\hline$C(22)-C(23)-C(24)$ & $119.8(3)$ \\
\hline $\mathrm{C}(22)-\mathrm{C}(23)-\mathrm{H}(23)$ & 120.1 \\
\hline $\mathrm{C}(24)-\mathrm{C}(23)-\mathrm{H}(23)$ & 120.1 \\
\hline$C(23)-C(24)-C(25)$ & $119.2(3)$ \\
\hline $\mathrm{C}(23)-\mathrm{C}(24)-\mathrm{H}(24)$ & 120.4 \\
\hline
\end{tabular}




\begin{tabular}{|c|c|}
\hline $\mathrm{C}(25)-\mathrm{C}(24)-\mathrm{H}(24)$ & 120.4 \\
\hline$C(26)-C(25)-C(24)$ & $119.9(3)$ \\
\hline $\mathrm{C}(26)-\mathrm{C}(25)-\mathrm{H}(25)$ & 120.1 \\
\hline $\mathrm{C}(24)-\mathrm{C}(25)-\mathrm{H}(25)$ & 120.1 \\
\hline$N(1)-C(26)-C(25)$ & $120.2(3)$ \\
\hline $\mathrm{N}(1)-\mathrm{C}(26)-\mathrm{H}(26)$ & 119.9 \\
\hline $\mathrm{C}(25)-\mathrm{C}(26)-\mathrm{H}(26)$ & 119.9 \\
\hline$C(33)-P(2)-C(27)$ & $106.33(12)$ \\
\hline$C(33)-P(2)-C(39)$ & $102.99(12)$ \\
\hline$C(27)-P(2)-C(39)$ & $102.23(12)$ \\
\hline$C(33)-P(2)-P d(1)$ & $114.32(9)$ \\
\hline$C(27)-P(2)-P d(1)$ & $112.99(9)$ \\
\hline$C(39)-P(2)-P d(1)$ & $116.62(8)$ \\
\hline$C(32)-C(27)-C(28)$ & $119.9(2)$ \\
\hline$C(32)-C(27)-P(2)$ & $119.6(2)$ \\
\hline$C(28)-C(27)-P(2)$ & $120.5(2)$ \\
\hline$C(29)-C(28)-C(27)$ & $120.2(3)$ \\
\hline $\mathrm{C}(29)-\mathrm{C}(28)-\mathrm{H}(28)$ & 119.9 \\
\hline $\mathrm{C}(27)-\mathrm{C}(28)-\mathrm{H}(28)$ & 119.9 \\
\hline$C(28)-C(29)-C(30)$ & $119.7(3)$ \\
\hline $\mathrm{C}(28)-\mathrm{C}(29)-\mathrm{H}(29)$ & 120.2 \\
\hline$C(30)-C(29)-H(29)$ & 120.2 \\
\hline$C(31)-C(30)-C(29)$ & $120.0(3)$ \\
\hline $\mathrm{C}(31)-\mathrm{C}(30)-\mathrm{H}(30)$ & 120.0 \\
\hline $\mathrm{C}(29)-\mathrm{C}(30)-\mathrm{H}(30)$ & 120.0 \\
\hline$C(30)-C(31)-C(32)$ & $120.7(3)$ \\
\hline $\mathrm{C}(30)-\mathrm{C}(31)-\mathrm{H}(31)$ & 119.7 \\
\hline $\mathrm{C}(32)-\mathrm{C}(31)-\mathrm{H}(31)$ & 119.7 \\
\hline$C(27)-C(32)-C(31)$ & $119.5(3)$ \\
\hline $\mathrm{C}(27)-\mathrm{C}(32)-\mathrm{H}(32)$ & 120.2 \\
\hline $\mathrm{C}(31)-\mathrm{C}(32)-\mathrm{H}(32)$ & 120.2 \\
\hline$C(38)-C(33)-C(34)$ & $119.0(2)$ \\
\hline$C(38)-C(33)-P(2)$ & $123.7(2)$ \\
\hline$C(34)-C(33)-P(2)$ & $117.2(2)$ \\
\hline$C(35)-C(34)-C(33)$ & $120.4(2)$ \\
\hline $\mathrm{C}(35)-\mathrm{C}(34)-\mathrm{H}(34)$ & 119.8 \\
\hline $\mathrm{C}(33)-\mathrm{C}(34)-\mathrm{H}(34)$ & 119.8 \\
\hline$C(36)-C(35)-C(34)$ & $120.3(3)$ \\
\hline $\mathrm{C}(36)-\mathrm{C}(35)-\mathrm{H}(35)$ & 119.9 \\
\hline $\mathrm{C}(34)-\mathrm{C}(35)-\mathrm{H}(35)$ & 119.9 \\
\hline$C(35)-C(36)-C(37)$ & $119.9(3)$ \\
\hline $\mathrm{C}(35)-\mathrm{C}(36)-\mathrm{H}(36)$ & 120.0 \\
\hline $\mathrm{C}(37)-\mathrm{C}(36)-\mathrm{H}(36)$ & 120.0 \\
\hline$C(38)-C(37)-C(36)$ & $120.2(3)$ \\
\hline $\mathrm{C}(38)-\mathrm{C}(37)-\mathrm{H}(37)$ & 119.9 \\
\hline $\mathrm{C}(36)-\mathrm{C}(37)-\mathrm{H}(37)$ & 119.9 \\
\hline$C(37)-C(38)-C(33)$ & $120.2(3)$ \\
\hline $\mathrm{C}(37)-\mathrm{C}(38)-\mathrm{H}(38)$ & 119.9 \\
\hline $\mathrm{C}(33)-\mathrm{C}(38)-\mathrm{H}(38)$ & 119.9 \\
\hline$C(44)-C(39)-C(40)$ & $118.9(2)$ \\
\hline$C(44)-C(39)-P(2)$ & $118.1(2)$ \\
\hline$C(40)-C(39)-P(2)$ & $122.9(2)$ \\
\hline$C(41)-C(40)-C(39)$ & $119.4(3)$ \\
\hline $\mathrm{C}(41)-\mathrm{C}(40)-\mathrm{H}(40)$ & 120.3 \\
\hline
\end{tabular}




\begin{tabular}{|c|c|}
\hline $\mathrm{C}(39)-\mathrm{C}(40)-\mathrm{H}(40)$ & 120.3 \\
\hline$C(42)-C(41)-C(40)$ & $121.4(3)$ \\
\hline $\mathrm{C}(42)-\mathrm{C}(41)-\mathrm{H}(41)$ & 119.3 \\
\hline $\mathrm{C}(40)-\mathrm{C}(41)-\mathrm{H}(41)$ & 119.3 \\
\hline$C(41)-C(42)-C(43)$ & $119.5(3)$ \\
\hline $\mathrm{C}(41)-\mathrm{C}(42)-\mathrm{H}(42)$ & 120.3 \\
\hline $\mathrm{C}(43)-\mathrm{C}(42)-\mathrm{H}(42)$ & 120.3 \\
\hline$C(44)-C(43)-C(42)$ & $119.8(3)$ \\
\hline $\mathrm{C}(44)-\mathrm{C}(43)-\mathrm{H}(43)$ & 120.1 \\
\hline$C(42)-C(43)-H(43)$ & 120.1 \\
\hline$C(43)-C(44)-C(39)$ & $121.0(3)$ \\
\hline $\mathrm{C}(43)-\mathrm{C}(44)-\mathrm{H}(44)$ & 119.5 \\
\hline $\mathrm{C}(39)-\mathrm{C}(44)-\mathrm{H}(44)$ & 119.5 \\
\hline $\mathrm{O}(3)-\mathrm{Cl}(2)-\mathrm{O}(5)$ & $113.4(2)$ \\
\hline$O(3)-\mathrm{Cl}(2)-\mathrm{O}(2)$ & $108.0(2)$ \\
\hline $\mathrm{O}(5)-\mathrm{Cl}(2)-\mathrm{O}(2)$ & $109.9(2)$ \\
\hline $\mathrm{O}(3)-\mathrm{Cl}(2)-\mathrm{O}(4)$ & $106.9(2)$ \\
\hline $\mathrm{O}(5)-\mathrm{Cl}(2)-\mathrm{O}(4)$ & $108.2(2)$ \\
\hline $\mathrm{O}(2)-\mathrm{Cl}(2)-\mathrm{O}(4)$ & $110.3(3)$ \\
\hline $\mathrm{Cl}(4)-\mathrm{C}(45)-\mathrm{Cl}(3)$ & $112.9(2)$ \\
\hline $\mathrm{Cl}(4)-\mathrm{C}(45)-\mathrm{H}(45 \mathrm{~A})$ & 109.0 \\
\hline $\mathrm{Cl}(3)-\mathrm{C}(45)-\mathrm{H}(45 \mathrm{~A})$ & 109.0 \\
\hline $\mathrm{Cl}(4)-\mathrm{C}(45)-\mathrm{H}(45 \mathrm{~B})$ & 109.0 \\
\hline $\mathrm{Cl}(3)-\mathrm{C}(45)-\mathrm{H}(45 \mathrm{~B})$ & 109.0 \\
\hline $\mathrm{H}(45 \mathrm{~A})-\mathrm{C}(45)-\mathrm{H}(45 \mathrm{~B})$ & 107.8 \\
\hline $\mathrm{Cl}(6)-\mathrm{C}(46)-\mathrm{Cl}(5)$ & $111.03(16)$ \\
\hline $\mathrm{Cl}(6)-\mathrm{C}(46)-\mathrm{H}(46 \mathrm{~A})$ & 109.4 \\
\hline $\mathrm{Cl}(5)-\mathrm{C}(46)-\mathrm{H}(46 \mathrm{~A})$ & 109.4 \\
\hline $\mathrm{Cl}(6)-\mathrm{C}(46)-\mathrm{H}(46 \mathrm{~B})$ & 109.4 \\
\hline $\mathrm{Cl}(5)-\mathrm{C}(46)-\mathrm{H}(46 \mathrm{~B})$ & 109.4 \\
\hline $\mathrm{H}(46 \mathrm{~A})-\mathrm{C}(46)-\mathrm{H}(46 \mathrm{~B})$ & 108.0 \\
\hline $\mathrm{Cl}(8)-\mathrm{C}(47)-\mathrm{Cl}(7)$ & $110.43(17)$ \\
\hline $\mathrm{Cl}(8)-\mathrm{C}(47)-\mathrm{H}(47 \mathrm{~A})$ & 109.6 \\
\hline $\mathrm{Cl}(7)-\mathrm{C}(47)-\mathrm{H}(47 \mathrm{~A})$ & 109.6 \\
\hline $\mathrm{Cl}(8)-\mathrm{C}(47)-\mathrm{H}(47 \mathrm{~B})$ & 109.6 \\
\hline $\mathrm{Cl}(7)-\mathrm{C}(47)-\mathrm{H}(47 \mathrm{~B})$ & 109.6 \\
\hline $\mathrm{H}(47 \mathrm{~A})-\mathrm{C}(47)-\mathrm{H}(47 \mathrm{~B})$ & 108.1 \\
\hline
\end{tabular}

Symmetry transformations used to generate equivalent atoms: 
Table 4. Anisotropic displacement parameters $\left[\AA^{2} \times 10^{3}\right]$ for $6 \cdot 3 \mathbf{C H}_{2} \mathrm{Cl}_{2}$. The anisotropic displacement factor exponent takes the form: $-2 \pi^{2}\left[\left(h a^{*}\right)^{2} U_{11}+\right.$ $\left.\ldots+2 h k a * b * U_{12}\right]$

\begin{tabular}{|c|c|c|c|c|c|c|}
\hline & $\mathrm{U} 11$ & $\mathrm{U} 22$ & U33 & $\mathrm{U} 23$ & U13 & $\mathrm{U} 12$ \\
\hline $\mathrm{Pd}(1)$ & $14(1)$ & $15(1)$ & $14(1)$ & $0(1)$ & $0(1)$ & $0(1)$ \\
\hline $\mathrm{Cl}(1)$ & $16(1)$ & $20(1)$ & $23(1)$ & $3(1)$ & $-2(1)$ & $0(1)$ \\
\hline C (1) & $17(1)$ & $18(1)$ & $20(1)$ & $1(1)$ & $-4(1)$ & $-2(1)$ \\
\hline$O(1)$ & $19(1)$ & $24(1)$ & $18(1)$ & $0(1)$ & $2(1)$ & $-2(1)$ \\
\hline C (2) & $16(1)$ & $17(1)$ & $16(1)$ & $-3(1)$ & $-1(1)$ & $-3(1)$ \\
\hline $\mathrm{P}(1)$ & $17(1)$ & $16(1)$ & $17(1)$ & $-2(1)$ & $0(1)$ & $-2(1)$ \\
\hline C (3) & $22(1)$ & $18(1)$ & $18(1)$ & $1(1)$ & $-2(1)$ & $-6(1)$ \\
\hline$C(4)$ & $27(1)$ & $28(2)$ & $20(1)$ & $-2(1)$ & $-1(1)$ & $-2(1)$ \\
\hline C (5) & $39(2)$ & $39(2)$ & $22(1)$ & $-5(1)$ & $-5(1)$ & $-2(2)$ \\
\hline$C(6)$ & $38(2)$ & $41(2)$ & $21(1)$ & $2(1)$ & $-11(1)$ & $-6(2)$ \\
\hline$C(7)$ & $28(2)$ & $33(2)$ & $31(2)$ & $7(1)$ & $-10(1)$ & $-3(1)$ \\
\hline$C(8)$ & $23(1)$ & $23(1)$ & $23(1)$ & $3(1)$ & $-3(1)$ & $-3(1)$ \\
\hline C (9) & $18(1)$ & $18(1)$ & $28(1)$ & $-2(1)$ & $1(1)$ & $-1(1)$ \\
\hline$C(10)$ & $28(2)$ & $29(2)$ & $30(2)$ & $1(1)$ & $0(1)$ & $-10(1)$ \\
\hline$C(11)$ & $35(2)$ & $32(2)$ & $43(2)$ & $4(1)$ & $7(1)$ & $-13(1)$ \\
\hline C (12) & $22(2)$ & $25(2)$ & $59(2)$ & $-5(2)$ & $8(1)$ & $-5(1)$ \\
\hline$C(13)$ & $23(1)$ & $22(1)$ & $47(2)$ & $-8(2)$ & $-4(2)$ & $-2(1)$ \\
\hline$C(14)$ & $23(1)$ & $20(1)$ & $32(2)$ & $-6(1)$ & $-3(1)$ & $0(1)$ \\
\hline$C(15)$ & $20(1)$ & $20(1)$ & $19(1)$ & $-2(1)$ & $-2(1)$ & $0(1)$ \\
\hline$C(16)$ & $25(1)$ & $21(1)$ & $25(1)$ & $-1(1)$ & $-2(1)$ & $-1(1)$ \\
\hline$C(17)$ & $27(2)$ & $22(1)$ & $33(2)$ & $-6(1)$ & $-3(1)$ & $2(1)$ \\
\hline$C(18)$ & $23(1)$ & $30(2)$ & $29(1)$ & $-9(1)$ & $-3(1)$ & $8(1)$ \\
\hline$C(19)$ & $22(1)$ & $34(2)$ & $27(1)$ & $-2(1)$ & $2(1)$ & $4(1)$ \\
\hline$C(20)$ & $22(1)$ & $20(1)$ & $30(1)$ & $0(1)$ & $3(1)$ & $1(1)$ \\
\hline C (21) & $14(1)$ & $18(1)$ & $17(1)$ & $-3(1)$ & $-3(1)$ & $-1(1)$ \\
\hline $\mathrm{N}(1)$ & $14(1)$ & $17(1)$ & $21(1)$ & $0(1)$ & $0(1)$ & $2(1)$ \\
\hline C (22) & $17(1)$ & $22(1)$ & $28(1)$ & $2(1)$ & $-3(1)$ & $0(1)$ \\
\hline C (23) & $19(1)$ & $22(2)$ & $45(2)$ & $0(1)$ & $-3(1)$ & $3(1)$ \\
\hline$C(24)$ & $21(1)$ & $27(2)$ & $50(2)$ & $-13(1)$ & $5(1)$ & $4(1)$ \\
\hline$C(25)$ & $24(1)$ & $30(2)$ & $33(2)$ & $-11(1)$ & $6(1)$ & $-3(1)$ \\
\hline$C(26)$ & $17(1)$ & $21(1)$ & $26(1)$ & $-5(1)$ & $2(1)$ & $-2(1)$ \\
\hline $\mathrm{P}(2)$ & $15(1)$ & $15(1)$ & $13(1)$ & $0(1)$ & $0(1)$ & $1(1)$ \\
\hline C (27) & $16(1)$ & $20(1)$ & $16(1)$ & $-3(1)$ & $-1(1)$ & $2(1)$ \\
\hline$C(28)$ & $22(1)$ & $20(1)$ & $19(1)$ & $-1(1)$ & $0(1)$ & $1(1)$ \\
\hline C (29) & $22(1)$ & $26(1)$ & $25(1)$ & $-9(1)$ & $-1(1)$ & $2(1)$ \\
\hline$C(30)$ & $29(2)$ & $33(2)$ & $15(1)$ & $-5(1)$ & $-1(1)$ & $9(1)$ \\
\hline C (31) & $26(1)$ & $34(2)$ & $15(1)$ & $4(1)$ & $3(1)$ & $4(1)$ \\
\hline C (32) & $19(1)$ & $23(1)$ & $17(1)$ & $1(1)$ & $0(1)$ & $0(1)$ \\
\hline C (33) & $12(1)$ & $19(1)$ & $19(1)$ & $4(1)$ & $-1(1)$ & $-1(1)$ \\
\hline$C(34)$ & $19(1)$ & $17(1)$ & $19(1)$ & $-1(1)$ & $1(1)$ & $0(1)$ \\
\hline C (35) & $22(1)$ & $27(2)$ & $18(1)$ & $2(1)$ & $-3(1)$ & $0(1)$ \\
\hline$C(36)$ & $29(2)$ & $24(2)$ & $27(1)$ & $6(1)$ & $-6(1)$ & $6(1)$ \\
\hline C (37) & $32(2)$ & $24(2)$ & $31(2)$ & $-4(1)$ & $-2(1)$ & $9(1)$ \\
\hline$C(38)$ & $24(1)$ & $21(1)$ & $23(2)$ & $-2(1)$ & $-1(1)$ & $4(1)$ \\
\hline C (39) & $18(1)$ & $17(1)$ & $15(1)$ & $-3(1)$ & $1(1)$ & $-3(1)$ \\
\hline$C(40)$ & $19(1)$ & $21(1)$ & $18(1)$ & $3(1)$ & $2(1)$ & $2(1)$ \\
\hline
\end{tabular}




\begin{tabular}{lrrrrrr}
$C(41)$ & $25(2)$ & $27(2)$ & $24(1)$ & $3(1)$ & $7(1)$ & $1(1)$ \\
$C(42)$ & $20(1)$ & $25(1)$ & $26(1)$ & $-5(1)$ & $7(1)$ & $-5(1)$ \\
$C(43)$ & $18(1)$ & $24(1)$ & $24(1)$ & $-5(1)$ & $0(1)$ & $-1(1)$ \\
$C(44)$ & $22(1)$ & $19(1)$ & $16(1)$ & $-1(1)$ & $-1(1)$ & $-1(1)$ \\
$C 1(2)$ & $30(1)$ & $31(1)$ & $19(1)$ & $-2(1)$ & $1(1)$ & $1(1)$ \\
$O(2)$ & $67(2)$ & $128(3)$ & $26(1)$ & $-18(2)$ & $-15(1)$ & $58(2)$ \\
$O(3)$ & $57(2)$ & $113(3)$ & $23(1)$ & $13(1)$ & $8(1)$ & $1(2)$ \\
$O(4)$ & $34(1)$ & $35(1)$ & $138(4)$ & $0(2)$ & $21(2)$ & $-2(1)$ \\
$O(5)$ & $76(2)$ & $34(2)$ & $87(2)$ & $-26(2)$ & $-23(2)$ & $14(2)$ \\
$C(45)$ & $64(3)$ & $40(2)$ & $60(2)$ & $8(2)$ & $13(2)$ & $-6(2)$ \\
$C 1(3)$ & $135(2)$ & $62(1)$ & $117(1)$ & $32(1)$ & $34(1)$ & $45(1)$ \\
$C 1(4)$ & $75(1)$ & $89(1)$ & $101(1)$ & $-16(1)$ & $26(1)$ & $-41(1)$ \\
$C(46)$ & $30(2)$ & $29(2)$ & $24(1)$ & $-2(1)$ & $-2(1)$ & $-6(1)$ \\
$C 1(5)$ & $27(1)$ & $32(1)$ & $43(1)$ & $-5(1)$ & $-6(1)$ & $0(1)$ \\
$C 1(6)$ & $30(1)$ & $32(1)$ & $26(1)$ & $-3(1)$ & $0(1)$ & $2(1)$ \\
$C(47)$ & $27(1)$ & $34(2)$ & $30(1)$ & $4(1)$ & $2(1)$ & $-2(1)$ \\
$C 1(7)$ & $31(1)$ & $37(1)$ & $48(1)$ & $-3(1)$ & $8(1)$ & $2(1)$ \\
$C l(8)$ & $25(1)$ & $39(1)$ & $29(1)$ & $3(1)$ & $-2(1)$ & $1(1)$ \\
& & & & & & \\
\hline
\end{tabular}


Table 5. Hydrogen coordinates $\left(\times 10^{4}\right)$ and isotropic displacement parameters $\left(\AA^{2} \times 10^{3}\right)$ for $6 \cdot 3 \mathrm{CH}_{2} \mathrm{Cl}_{2}$.

\begin{tabular}{|c|c|c|c|c|}
\hline & $\mathrm{x}$ & y & z & $\mathrm{U}(\mathrm{eq})$ \\
\hline H (2) & 7742 & 6628 & 8094 & 20 \\
\hline $\mathrm{H}(4)$ & 7793 & 6611 & 3926 & 30 \\
\hline H (5) & 7185 & 6464 & 1954 & 40 \\
\hline $\mathrm{H}(6)$ & 6154 & 6892 & 1620 & 40 \\
\hline H ( 7) & 5694 & 7444 & 3254 & 37 \\
\hline H ( 8) & 6288 & 7597 & 5242 & 28 \\
\hline $\mathrm{H}(10)$ & 8106 & 7802 & 4134 & 35 \\
\hline $\mathrm{H}(11)$ & 9151 & 8223 & 3770 & 44 \\
\hline $\mathrm{H}(12)$ & 9986 & 8198 & 5412 & 42 \\
\hline $\mathrm{H}(13)$ & 9776 & 7775 & 7463 & 37 \\
\hline $\mathrm{H}(14)$ & 8725 & 7362 & 7831 & 30 \\
\hline $\mathrm{H}(16)$ & 7520 & 8305 & 6988 & 28 \\
\hline $\mathrm{H}(17)$ & 6913 & 8834 & 8499 & 33 \\
\hline $\mathrm{H}(18)$ & 6065 & 8449 & 9809 & 33 \\
\hline $\mathrm{H}(19)$ & 5815 & 7534 & 9601 & 33 \\
\hline $\mathrm{H}(20)$ & 6440 & 6997 & 8120 & 28 \\
\hline $\mathrm{H}(21)$ & 8242 & 5743 & 8230 & 20 \\
\hline $\mathrm{H}(22)$ & 8975 & 5070 & 8515 & 27 \\
\hline H (23) & 9710 & 4417 & 7736 & 35 \\
\hline H (24) & 9808 & 4265 & 5447 & 39 \\
\hline $\mathrm{H}(25)$ & 9157 & 4779 & 3971 & 35 \\
\hline $\mathrm{H}(26)$ & 8420 & 5428 & 4793 & 25 \\
\hline $\mathrm{H}(28)$ & 6265 & 4003 & 5303 & 24 \\
\hline H (29) & 6193 & 3694 & 3104 & 29 \\
\hline H (30) & 6677 & 4199 & 1375 & 31 \\
\hline $\mathrm{H}(31)$ & 7217 & 5007 & 1844 & 30 \\
\hline H (32) & 7279 & 5326 & 4037 & 24 \\
\hline H (34) & 7428 & 4920 & 9166 & 22 \\
\hline H (35) & 8087 & 4331 & 10415 & 27 \\
\hline $\mathrm{H}(36)$ & 8465 & 3528 & 9483 & 32 \\
\hline H (37) & 8203 & 3321 & 7266 & 35 \\
\hline $\mathrm{H}(38)$ & 7543 & 3909 & 5998 & 27 \\
\hline $\mathrm{H}(40)$ & 6314 & 4326 & 8898 & 23 \\
\hline H ( 41) & 5222 & 4134 & 9653 & 30 \\
\hline $\mathrm{H}(42)$ & 4280 & 4429 & 8501 & 28 \\
\hline H ( 43) & 4418 & 4969 & 6599 & 26 \\
\hline H ( 44$)$ & 5501 & 5168 & 5831 & 23 \\
\hline $\mathrm{H}(45 \mathrm{~A})$ & 8551 & 7130 & 2135 & 65 \\
\hline $\mathrm{H}(45 \mathrm{~B})$ & 8613 & 6926 & 628 & 65 \\
\hline $\mathrm{H}(46 \mathrm{~A})$ & 5333 & 5537 & 9500 & 33 \\
\hline $\mathrm{H}(46 \mathrm{~B})$ & 5592 & 5889 & 8263 & 33 \\
\hline $\mathrm{H}(47 \mathrm{~A})$ & 10403 & 6738 & 6837 & 36 \\
\hline $\mathrm{H}(47 \mathrm{~B})$ & 9717 & 6552 & 6115 & 36 \\
\hline
\end{tabular}

